\title{
Bystander Effect Studies in HL60 Human Promyelocytes
}

V. Dini, M. Belli, O. Sapora, G. Simone, E. Sorrentino and M.A. Tabocchini

Istituto Superiore di Sanità EJ INFN Gr coll Sanità, Viale Regina Elena 299, 00161 Rome,

Italy

v.dini@iss.it

In the framework of the NOTE Integrated Project (FP6-36465, Euratom), we used for the first time HL60 human promyelocytes for bystander effects studies. This cell line is capable to differentiate in vitro towards either granulocytes or monocytes/macrophages and, therefore, one of the major interest is that it is possible to investigate the nature of signals released from the same cell type in various differentiating conditions and also to evaluate if cell differentiation can be induced through bystander mechanisms. The experiments have been focused (i) to the characterization of the biological system using the appropriate cluster of differentiation (CD) of cell surface molecules for the different cells of interest (i.e., promyelocytes, monocyte-and macrophage-like cells) and (ii) to obtain information about the response to signalling factors from sham/irradiated HL60 promyelocytes in terms of micronuclei (MN) induction and cell killing. Experiments were also performed to characterize the basal levels of ROS and RNS in cells at different stages of differentiation. Monocyte- and macrophage-like differentiation was obtained using vitamin D3 and phorbol diester (12-O-tetradecanoylphorbol-13acetate, TPA) as inducers, respectively. Irradiation was performed with gamma rays and bystander experiments were carried out using the medium transfer approach. The experiments performed to characterize the differentiation status in the HL60 clone we used show that CD11b and CD11c appear both suitable for identifying macrophage-like and monocyte-like cells. The former are negative while the latter are positive to CD14. Actively proliferating promyelocytes present some positivity to CD95 but not to CD11b and CD11c or CD14. At the present experiments are planned aimed at distinguishing promyelocytes from macrophage-like cells in a mixed population. The results on MN induction in unirradiated promyelocytes by the medium collected from promyelocytes irradiated with 0.5 Gy and incubated for different times at $37^{\circ} \mathrm{C}$ show an increase after $2 \mathrm{~h}$ incubation that disappears at longer incubation times. Measurements of cell killing show that incubation with "conditioned" medium from 1 Gy irradiated promyelocytes leads to an increase in the cloning efficiency of the bystander promyelocytes, giving surviving fractions higher than 1 . However, this effect seems more related to factors physiologically released by the cells than to factors induced by irradiation, as indicated by the comparison with medium from sham-irradiated cells. These results suggest that growth stimulating factors, instead of growth inhibiting factors, are released by HL60 promyelocytes. 